ALEA, Lat. Am. J. Probab. Math. Stat. 16, 855-870 (2019)

DOI: 10.30757/ALEA.v16-31

\title{
Cone exponents of Brownian motions with particular drifts
}

\section{Izumi Okada}

Kyushu university, Motooka 744, Nishi-ku, Fukuoka, 819-0395, Japan.

E-mail address: iokada@math.kyushu-u.ac.jp

\begin{abstract}
Herein, we describe multidimensional Brownian motions for $d=2,3$ with drifts whose order is the same as that of the mean displacement of a Brownian motion. We consider the probabilities that the processes remain in specific cones for a considerable amount of time. We obtain exponents expressing the probabilities, which are different from that of the ordinary Brownian motion. Finally, we suggest an open problem concerning the exact values.
\end{abstract}

\section{Introduction and Known Results}

Herein, we describe Brownian motions with drifts $B_{t}+F_{t}$ whose order is same as that of the mean displacement of a Brownian motion $\left(B_{t}\right)_{t \geq 0}$. Although the topic is classical, multiple things have been remained and under investigation. A previous study (Antunović et al., 2011) considered the standard one-dimensional Brownian motion $B_{t}$ with $\alpha$-Hölder continuous functions $F_{t}$ with $\alpha \leq 1 / 2$. Another study (Peres and Sousi, 2012) showed that the hitting time satisfies the intersection equivalence concerning standard one-dimensional and multidimensional Brownian motions with 1/2-Hölder continuous functions. Furthermore, previous research (Mörters and Peres, 2010) proposed several other related open problems. Herein, we investigate the probabilities that the processes remain in specific cones for considerable amount of time. In particular, we show that the probabilities satisfy submultiplicativity and supermultiplicativity. This indicates that the probabilities are of polynomial order. In addition, it shows the existence of cone exponents $\xi$ for the processes. In other words, the probability that a Brownian motion with specific drifts remains in a specific cone until time $n^{2}$ is asymptotically close to $n^{-\xi}$. Our results show that the cone exponents are different from those of the ordinary Brownian motion, and they are positive and finite.

Received by the editors September 14th, 2018; accepted May 23th, 2019.

2010 Mathematics Subject Classification. 60J25.

Key words and phrases. Simple random walk, local time. 
Next, we introduce the details of known results concerning the cone estimates of the Brownian motion. If

$$
\begin{aligned}
& \beta=\left(\cos \beta_{1}, \sin \beta_{1}\right) \quad(d=2), \\
& \beta=\left(\cos \beta_{1} \cos \beta_{2}, \sin \beta_{1} \cos \beta_{2}, \sin \beta_{2}\right) \quad(\mathrm{d}=3)
\end{aligned}
$$

for some $0 \leq \beta_{1}, \beta_{2}<2 \pi$, let $\arg (\beta, b)$ be the angle between the line connecting the origin and $\beta$ and the line connecting the origin and $b$. For $d=2,3,0<\alpha<\pi$, and $0 \leq \beta_{1}, \beta_{2}<2 \pi$, define the open cone $W[\alpha, \beta]:=\left\{z \in \mathbb{R}^{d}: \arg (\beta, z)<\alpha / 2\right\}$. Let $\{B(t)\}_{t \geq 0}$ be a Brownian motion for $d=2,3$. We denote the Brownian trajectory for $T \subset \mathbb{R}^{+} \cup\{0\}$ by $B[T]$. For $d=2$, we define $C_{t}^{+}(\alpha)$ (resp., $C_{t}^{-}(\alpha)$ ) as a set of times that $B$ spends in $W[\alpha, \beta]$ with some $\beta$, i.e.,

$$
\begin{aligned}
& C_{t}^{+}(\alpha):=\cup_{\beta \in[0,2 \pi]}\{t \in[0,1): \exists h>0, B(s) \in B(t)+W[\alpha, \beta], s \in[t, t+h]\}, \\
& C_{t}^{-}(\alpha):=\cup_{\beta \in[0,2 \pi]}\{t \in[0,1): \exists h>0, B(s) \in B(t)+W[\alpha, \beta], s \in[t-h, t]\} .
\end{aligned}
$$

The corresponding points of the Brownian trajectory are called $\alpha$-cone points. We let the set of $\alpha$-cone points be $C_{p}^{+}(\alpha)$ or $C_{p}^{-}(\alpha)$, i.e.,

$$
\begin{aligned}
& C_{p}^{+}(\alpha):=\cup_{\beta \in[0,2 \pi]}\left\{B_{t}: t \in[0,1) \exists h>0, B(s) \in B(t)+W[\alpha, \beta], s \in[t, t+h]\right\}, \\
& C_{p}^{-}(\alpha):=\cup_{\beta \in[0,2 \pi]}\left\{B_{t}: t \in[0,1) \exists h>0, B(s) \in B(t)+W[\alpha, \beta], s \in[t-h, t]\right\} .
\end{aligned}
$$

It was shown

$$
\begin{aligned}
\operatorname{dim}\left(C_{p}^{+}(\alpha) \cap C_{p}^{-}(\alpha)\right) & =2-\frac{2 \pi}{\alpha} \quad \text { a.s. } \\
\operatorname{dim}\left(C_{p}^{+}(\alpha)\right) & =2-\frac{\pi}{\alpha} \quad \text { a.s. }
\end{aligned}
$$

in Evans (1985). Another proof was given in Mörters and Peres (2010, Section 10.4). Burdzy (1985) and Shimura (1985) independently showed that one-sided cone points with angle $\alpha$ exist when $\alpha<\pi / 4$. Le Gall and Meyre (1992) showed that a cone point with angle $\pi / 4$ does not exist. Lawler (1999, Section 3) argued with cone exponents, which describe the probability that the Brownian motion stays in a cone. For $d=2$, the value of cone exponents were obtained (see Mörters and Peres, 2010, Section 10.4). Moreover, in a previous study (Garbit and Raschel, 2014), the tail distribution of the first exit time from a typical cone was observed for a Brownian motion with a drift of order $t$. The results of Garbit and Raschel (2014) are not the same as those of this paper since the order of the drift in this paper is different from that in Garbit and Raschel (2014). In addition, there are several order results that deal with other rear points e.g., slow points, where the Brownian motion is slow in Barlow and Perkins (1986); Davis and Perkins (1985). The following results are not directly connected but important.

\section{Main Results}

To state the principal results, we introduce the following notation. Let $d=2$, 3. For $x \in \mathbb{R}^{d}$ and $r>0$, let $\mathcal{B}(x, r):=\left\{y \in \mathbb{R}^{d}:|x-y| \leq r\right\}$. For $A \subset \mathbb{R}^{d}$, let $\partial A$ be the boundary of $A$. In particular, we write $\mathcal{B}_{r}$ for $\mathcal{B}(0, r)$ and $\mathcal{B}$ for $\mathcal{B}_{1}$. For any $x=\left(x_{1}, \ldots, x_{d}\right) \in \mathbb{R}^{d}$, we denote $\left(n x_{1}, \ldots, n x_{d}\right)\left(\operatorname{resp} .,\left(x_{1} / n, \ldots, x_{d} / n\right)\right)$ by $n x($ resp., $x / n)$. For any $x \in \mathbb{R}^{d}$ and $A \subset \mathbb{R}^{d}$, let $x+A:=\left\{x+y \in \mathbb{R}^{d}: y \in A\right\}$. Let $F_{l}(t):=\left(K(t+l)^{1 / 2}-K l^{1 / 2}, 0\right)(d=2)$ and $F_{l}(t):=\left(K(t+l)^{1 / 2}-K l^{1 / 2}, 0,0\right)$ $(d=3)$ for $l \geq 0$. Let $\tilde{W}[\alpha, \beta]:=W[\alpha / 2, \beta]$. Let $P^{x}$ denote the probability of a 
Brownian motion starting at $x$. For $l \geq 0$, let $\tau_{n, l}:=\inf \left\{t>0:\left(B+F_{l}\right)(t) \in \partial \mathcal{B}_{n}\right\}$. For typographical reasons, we write $\tau(n, l)$ and $\mathcal{B}(r)$ et al. for $\tau_{n, l}$ and $\mathcal{B}_{r}$ et al, respectively. Let

$$
a_{n, l}:=\sup _{x \in \partial \mathcal{B}} P^{x}\left(\left(B+F_{l}\right)\left[0, \tau\left(e^{n}, l\right)\right] \subset W[\alpha, \beta]\right) .
$$

In addition, we write $a(n, l)$ for $a_{n, l}$. We write $F(t), a_{n}$, and $\tau_{n}$ for $F_{0}(t), a_{n, 0}$, and $\tau_{n, 0}$, respectively. In the following theorems, we consider the long-time behavior of $a_{n}$. Without loss of generality, we consider the function $F_{l}(t)$ that has a one-way drift. Now, we fix $K \geq 0, \alpha$, and $\beta$ unless otherwise stated. We occasionally omit the symbols $K, \alpha$ and $\beta$. Hereafter, the values $C$ and $c$ might vary from place to place.

We now state the principal results.

Theorem 2.1. For any $K<\infty, \alpha$ and $\beta$, there exists $C<\infty$ such that for any $n, m \in \mathbb{N}$

$$
a_{n+m} \leq C a_{n} a_{m}
$$

Theorem 2.2. For any $K<\infty, \alpha$ and $\beta$, there exists $C<\infty$ such that for any $n, m \in \mathbb{N}$

$$
a_{n} a_{m} \leq C a_{n+m} .
$$

Remark 2.3. In fact, we have stronger claims. If we fix $K_{0}>0$, we can uniformly choose $C$ in $K \in\left[0, K_{0}\right]$ in Theorems 2.1 and 2.2. We omit the proof since we can uniformly choose any constants $(C$ or $c)$ in $K \in\left[0, K_{0}\right]$ in the proofs of Theorems 2.1 and 2.2.

Corollary 2.4. There exist $c>0, C<\infty$ and $0<\xi(K, \alpha, \beta)<\infty$ such that for any $n \in \mathbb{N}$ and $x \in \tilde{W} \cap \partial \mathcal{B}$

$$
c n^{-\xi} \leq P^{x}\left((B+F)\left[0, \tau_{n}\right] \subset W[\alpha, \beta]\right)
$$

and for any $n \in \mathbb{N}$ and $x \in W \cap \partial \mathcal{B}$

$$
P^{x}\left((B+F)\left[0, \tau_{n}\right] \subset W[\alpha, \beta]\right) \leq C n^{-\xi} .
$$

Corollary 2.5. It holds that $\xi(K, \alpha, \beta) \rightarrow \xi(0, \alpha, \beta)$ as $K \rightarrow 0$. If $(1,0)($ or $(1,0,0)) \in W[\alpha, \beta]$, then $\xi(K, \alpha, \beta) \rightarrow 0$ as $K \rightarrow \infty$. If $(1,0)($ or $(1,0,0)) \notin \bar{W}[\alpha, \beta]$, then $\xi(K, \alpha, \beta) \rightarrow \infty$ as $K \rightarrow \infty$, where $\bar{A}$ denotes the closure of $A$.

Corollary 2.6. There exist $0<C_{1}, C_{2}<\infty$ such that for any $n \in \mathbb{N}$ and $x \in$ $\tilde{W} \cap \partial \mathcal{B}$,

$$
C_{1} n^{-\xi} \leq P^{x}\left((B+F)\left[0, n^{2}\right] \subset W[\alpha, \beta]\right) \leq C_{2} n^{-\xi} .
$$

Remark 2.7. Let $L_{k}:=\{t:(B+F)[0, t) \cap(B+F)(t, 1]=\emptyset\}$. The following result is obtained by an argument same as that in proof of Lawler (1996, Theorem 1.1) using Lawler (1996, Proposition 3.15) instead of Corollary 2.6:

$$
\operatorname{dim}_{h}\left(L_{k}\right)=1-\frac{\xi(K)}{2},
$$

where $\operatorname{dim}_{h}$ denotes the Hausdorff dimension.

Open Problem 2.8. We suggest an open problem concerning the exact values of $\xi(K, \alpha, \beta)$ for any $K>0,0<\alpha<\pi$, and $\beta$. 
Now, we provide proofs of the principal results. It is worth nothing that while Theorem 2.1 for $K=0$ (ordinary Brownian motion) is trivial using scaling and the Markov property, for $K>0$ (Brownian motion with drift) is not. Concretely, while $\left\{B(s): 0 \leq s \leq \tau_{n, 0}, B(0)=x\right\}={ }_{d}\left\{B\left(n^{2} s\right) / n: 0 \leq s \leq \tau_{n+m, 0}, B(0)=\right.$ $n x\}$ for $x \in \partial \mathcal{B}$, the corresponding result for a Brownian motion with drifts does not hold. Hence, we consider the subsidiary properties in Section 3.1 to show a result which close to that obtained in Theorem 2.1, i.e., the submultiplicativity of $\sup _{0 \leq l \leq 1} a_{n, l}$. In addition, we show a particular equivalence of $\sup _{0 \leq l \leq 1} a_{n, l}$ and $a_{n}$ using geometric structures of the cone and hence obtain the desired result.

\section{Proofs}

3.1. Preparations for principal results. In this section, we provide the properties of Brownian motions with drifts that correspond to the elementary properties of the ordinary Brownian motion.

Lemma 3.1. (The scaling property of Brownian motions with drifts)

Let $e$ be Napier's constant. It holds that for any $r>0$ and $x \in \mathbb{R}^{d}$

$$
\begin{aligned}
&\left\{\left(B+F_{l}\right)(s): 0 \leq s \leq r,\left(B+F_{l}\right)(0)=x\right\} \\
&={ }_{d}\left\{e\left(B+F_{l / e^{2}}\right)\left(s / e^{2}\right): 0 \leq s \leq r,\left(B+F_{l / e^{2}}\right)(0)=x / e\right\}
\end{aligned}
$$

and for any $r>0$ and $x \in \mathcal{B}_{r}$

$$
\begin{aligned}
&\left\{\left(B+F_{l}\right)(s): 0 \leq s \leq \tau_{r, l},\left(B+F_{l}\right)(0)=x\right\} \\
&={ }_{d}\left\{e\left(B+F_{l / e^{2}}\right)\left(s / e^{2}\right): 0 \leq s \leq \tau\left(r / e, l / e^{2}\right),\left(B+F_{l / e^{2}}\right)(0)=x / e\right\} .
\end{aligned}
$$

In particular, it holds that for any $l \geq 0, r>0$ and $x \in W \cap \mathcal{B}_{r}$

$$
P^{x}\left(\left(B+F_{l}\right)\left[0, \tau_{r, l}\right] \subset W\right)=P^{x / e}\left(\left(B+F_{l / e^{2}}\right)\left[0, \tau\left(r / e, l / e^{2}\right)\right] \subset W\right)
$$

and

$$
P^{x}\left(\left(B+F_{l}\right)[0, r] \subset W\right)=P^{x / e}\left(\left(B+F_{l / e^{2}}\right)\left[0, r / e^{2}\right] \subset W\right) .
$$

Proof: Note that for any $0<s, s^{\prime}<\infty$,

$$
F_{l}(s)-F_{l}\left(s^{\prime}\right)=e\left(F_{l / e^{2}}\left(s / e^{2}\right)-F_{l / e^{2}}\left(s^{\prime} / e^{2}\right)\right) .
$$

Then, the scaling property of the ordinary Brownian motion yields the desired results.

Lemma 3.2. (The (strong) Markov property of Brownian motions with drifts)

The (strong) Markov property of Brownian motions with drifts holds. For example, it holds that for any $l \geq 0, x \in \partial \mathcal{B}$ and $1<r, r^{\prime}<\infty$,

$$
\begin{aligned}
& P^{x}\left(\left(B+F_{l}\right)\left[0, \tau_{r+r^{\prime}, l}\right] \subset W\right) \\
= & E^{x}\left[P^{B\left(\tau_{r, l}\right)}\left(\left(B+F_{l}\right)\left[0, \tau_{r+r^{\prime}, l}\right] \subset W \mid \mathcal{F}\left(\tau_{r, l}\right)\right) 1\left\{\left(B+F_{l}\right)\left[0, \tau_{r, l}\right] \subset W\right\}\right] .
\end{aligned}
$$

Proof: The (strong) Markov property of the ordinary Brownian motion yields naturally the desired result.

Lemma 3.3. (The large deviation estimate of Brownian motions with drifts)

It holds that there exist $C<\infty$ and $c>0$ such that for any $n \in \mathbb{N}$ and $s>0$

$$
\sup _{l \geq 0} \sup _{x \in \partial \mathcal{B}(\exp (n-1))} P^{x}\left(\tau\left(e^{n}, l\right) \geq s e^{2 n}\right) \leq C \exp (-c s),
$$


and

$$
\sup _{l \geq 0} \sup _{x \in \mathcal{B}(\exp (n-1))} P^{x}\left(\tau\left(e^{n}, l\right) \leq s e^{2 n}\right) \leq C \exp (-c / s) .
$$

Proof: By the scaling property, it suffices to show the result for $n=1$. First we show (3.1). Let $\hat{\tau}_{n}:=\inf \left\{t>0: B(t) \in \partial \mathcal{B}_{n}\right\}$. Note that given that $\max _{l \geq 0} K\left(\left(K^{2} / 100\right) \wedge\left(100 K^{2}\right)^{-1}+l\right)^{1 / 2}-K l^{1 / 2} \leq 1 / 10$

$$
\begin{aligned}
\inf _{l \geq 0} \inf _{x \in \mathcal{B}(e)} P^{x}\left(\tau_{e, l}<1\right) & \geq \inf _{l \geq 0} \inf _{x \in \mathcal{B}(e)} P^{x}\left(\tau_{e, l}<\frac{K^{2}}{100} \wedge \frac{1}{100 K^{2}}\right) \\
& \geq \inf _{x \in \mathcal{B}(e)} P^{x}\left(\hat{\tau}_{e-1 / 10}<\frac{K^{2}}{100} \wedge \frac{1}{100 K^{2}}\right)>0 .
\end{aligned}
$$

Thus, there exist $C<\infty$ and $c>0$ such that for any $n \in \mathbb{N}$ and $s>0$

$$
\begin{aligned}
\sup _{l \geq 0} \sup _{x \in \partial \mathcal{B}} P^{x}\left(\tau_{e, l} \geq s\right) & \leq \sup _{l \geq 0} \sup _{x \in \partial \mathcal{B}} P^{x}\left(\tau_{e, l} \geq\lceil s\rceil-1\right) \\
& \leq\left(\sup _{l \geq 0} \sup _{x \in \mathcal{B}(e)} P^{x}\left(\tau_{e, l} \geq 1\right)\right)^{\lceil s\rceil-1} \leq C \exp (-c s)
\end{aligned}
$$

and hence the desired result holds. Next, we show (3.2). It suffices to show the result for all sufficiently small $s>0$ with $K s^{1 / 2} \leq 1$. The large deviation estimate of the ordinary Brownian motion (see Lawler, 1996, (11)) indicates that $C<\infty$ and $c>0$ exist such that for any $n \in \mathbb{N}$ and $s>0$,

$$
\begin{aligned}
\sup _{l \geq 0} \sup _{x \in \partial \mathcal{B}} P^{x}\left(\tau_{e, l} \leq s\right) & \leq \sup _{x \in \partial \mathcal{B}} P^{x}\left(\hat{\tau}_{e-K s^{1 / 2}} \leq s\right) \\
& \leq \sup _{x \in \partial \mathcal{B}} P^{x}\left(\hat{\tau}_{e-1} \leq s\right) \\
& \leq C \exp (-c / s),
\end{aligned}
$$

and hence, we obtain the desired results.

3.2. Proof of Theorem 2.1. To show Theorem 2.1, we introduce some lemmas. To begin, we let $\mathcal{G}_{0}:=\left[0, e^{2}\right]$ and $\mathcal{G}_{s}:=\left[e^{2 s}, e^{2(s+1)}\right]$. Let $A_{n, l}$ be the event such that $\left(B+F_{l}\right)\left[0, \tau\left(e^{n}, l\right)\right] \subset W$. In particular, we write $A_{n}$ for $A_{n, 0}$.

Lemma 3.4. There exists $0<a<\infty$ such that for any $n \in \mathbb{N}$

$$
\inf _{x \in \tilde{W} \cap \partial \mathcal{B}} \inf _{l \geq 0} P^{x}\left(A_{n, l}\right) \geq e^{-n a} .
$$

Proof: Let $\hat{W}[\alpha, \beta]:=W[\alpha / 4, \beta]$ and $W^{0}[\alpha, \beta]:=W[2 \alpha / 3, \beta]$. Pick $\epsilon>0$ such that $\epsilon<\operatorname{dist}\left(\partial W^{0}[\alpha, \beta] \cap \partial \mathcal{B}_{1 / 2}, \partial W \cap \partial \mathcal{B}_{1 / 2}\right)$. Then, we have

$$
0<\inf _{x \in \tilde{W} \cap \partial \mathcal{B}} P^{x}\left(B\left[0, \hat{\tau}_{e+\epsilon}\right] \subset W^{0}[\alpha, \beta], \hat{\tau}_{e+\epsilon}<K^{-2} \epsilon^{2} \wedge \hat{\tau}_{1 / 2}, B\left(\hat{\tau}_{e+\epsilon}\right) \in \hat{W}\right) .
$$

Note that for any $\epsilon>0$ it holds that $\max _{l \geq 0} F_{l}\left(K^{-2} \epsilon^{2}\right)=\epsilon$. For any $x \in \tilde{W} \cap \partial \mathcal{B}$

$$
\begin{aligned}
& P^{x}\left(B\left[0, \hat{\tau}_{e+\epsilon}\right] \subset W^{0}[\alpha, \beta], \hat{\tau}_{e+\epsilon}<K^{-2} \epsilon^{2} \wedge \hat{\tau}_{1 / 2}, B\left(\hat{\tau}_{e+\epsilon}\right) \in \hat{W}\right) \\
\leq & P^{x}\left(\cap_{l>0}\left(A_{1, l} \cap\left\{\left(B+F_{l}\right)\left(\tau_{e, l}\right) \in \tilde{W}\right\}\right)\right) .
\end{aligned}
$$

Then, we have

$$
\begin{gathered}
\inf _{x \in \tilde{W} \cap \partial \mathcal{B}} \inf _{l \geq 0} P^{x}\left(A_{1, l} \cap\left\{\left(B+F_{l}\right)\left(\tau_{e, l}\right) \in \tilde{W}\right\}\right) \\
\geq \inf _{x \in \tilde{W} \cap \partial \mathcal{B}} P^{x}\left(\cap_{l>0}\left(A_{1, l} \cap\left\{\left(B+F_{l}\right)\left(\tau_{e, l}\right) \in \tilde{W}\right\}\right)\right)>0,
\end{gathered}
$$


and hence,

$$
a:=-\log \inf _{x \in \tilde{W} \cap \partial \mathcal{B}} \inf _{l \geq 0} P^{x}\left(A_{1, l} \cap\left\{\left(B+F_{l}\right)\left(\tau_{e, l}\right) \in \tilde{W}\right\}\right)<\infty .
$$

Therefore, the strong Markov property yields

$$
\inf _{x \in \tilde{W} \cap \partial \mathcal{B}} \inf _{l \geq 0} P^{x}\left(A_{n, l}\right) \geq e^{-a} \inf _{x \in \tilde{W} \cap \partial \mathcal{B}(e)} \inf _{l \geq 0} P^{x}\left(A_{n, l}\right) .
$$

By iterating it, the scaling property yields the desired result.

To state the next lemma, let $L_{n, l}$ be the event that $\left(B+F_{l}\right)\left(\tau\left(e^{n}, l\right)\right) \in \tilde{W}$ and $\left(B+F_{l}\right)\left[0, \tau\left(e^{n}, l\right)\right] \subset W$.

Lemma 3.5. There exists $C>0$ such that for any $n \in \mathbb{N}$,

$$
\inf _{y \in \tilde{W} \cap \partial \mathcal{B}(\exp (n-1))} \inf _{l \geq 0} P^{x}\left(L_{n, l} \mid A_{n, l}\right) \geq C .
$$

Proof: Based on the scaling property, the desired result is obtained when the result for $n=1$ is proved. Let $D:=\operatorname{dist}(\partial W, y)$ and $u_{n}:=\sum_{j=n}^{\infty} j^{2} 2^{-j}$. For all sufficiently large $n \in \mathbb{N}$ such that $u_{n} \leq 1 / 4$, let $h_{n}$ be the infimum of

$$
\frac{P^{y}\left(L_{1, l}\right)}{P^{y}\left(A_{1, l}\right)}
$$

where the infimum is over $0 \leq r \leq u_{n}, l \geq 0$ and $y$ such that $D \geq 2^{-n}$ and $|y|=1+r$ for some $r>0$. The lemma will follow if we prove that $\inf _{n} h_{n}>0$. Then, it suffices to show that $h_{n}>0$ for each $n \in \mathbb{N}$ and that there exists a summable sequence $\delta_{n}<1$ such that $h_{n+1} \geq h_{n}\left(1-\delta_{n}\right)$. We first show $h_{n}>0$ for any $n \in \mathbb{N}$. Note that we can find the following infinite cone $\mathcal{O}$ based on an argument same as those in the separation lemma in Lawler (1995); Lawler and Vermesi (2012) and in Lawler (1999, Section 3),

$$
\begin{aligned}
& \text { (1) } \frac{D}{100} \leq|z-y| \leq \frac{D}{20} \\
& \text { (2) } y \in z+\mathcal{O}, \quad \frac{D}{100} \leq|y-\partial(z+\mathcal{O})| \leq \frac{D}{20} \\
& (3)(z+\mathcal{O}) \cap \mathcal{B} \subset \mathcal{B}\left(y, \frac{D}{10}\right), \\
& \text { (4)if } V:=(z+\mathcal{O}) \cap \mathcal{B}_{\exp (1 / 16)}^{c}, \text { then } \operatorname{dist}(\partial W, V) \geq \frac{\alpha}{10000} .
\end{aligned}
$$

With the aid of (1) (2) and (3), by Lemma 3.4 and the scaling property, there exist $C<\infty$ and $0<a<\infty$ such that for any $l \geq 0$,

$$
P^{y}\left(\left(B+F_{l}\right)\left[0, \tau_{\exp (1 / 16), l}\right] \subset z+\mathcal{O}\right) \geq C\left(\frac{c+\exp (1 / 16)}{D / 100}\right)^{-a} \geq C D^{a} .
$$

Thus, with the aid of (4), the strong Markov property yields that

$$
P^{y}\left(L_{1, l}\right) \geq P^{y}\left(\left(B+F_{l}\right)\left[0, \tau_{\exp (1 / 16), l}\right] \subset z+\mathcal{O}\right) \underset{x \in(z+\mathcal{O}) \cap \partial \mathcal{B}_{\exp (1 / 16)}}{\times} P^{x}\left(L_{1, l}\right) \geq C D^{a} .
$$

Hence, $h_{n}>0$. 
Next, we show the second claim. Assume that $0 \leq r \leq u_{n+1}$ and $D \geq 2^{-n-1}$. Let

$$
\begin{aligned}
w(j) & :=(1+r)+4 j 2^{-n}, D_{j, l}:=\operatorname{dist}\left(\left(B+F_{l}\right)(\tau(w(j), l)), W\right), \\
\rho_{l}^{\prime} & :=\inf \left\{j \geq 1: D_{j, l} \geq 2^{-n}\right\} \text { and } \rho_{l}:=\min \left(\rho_{l}^{\prime}, \frac{n^{2}}{4}\right) .
\end{aligned}
$$

Let $C_{j+1, n, l}$ be an event such that $D_{j+1, l} \geq 2^{-n}$. There exists $c>0$ such that the probability that $C_{j+1, n, l}$, starting from a given distribution in $\partial \mathcal{B}(w(j))$, is at least $c$ for any $l \geq 0$ and $j$. Iterating this, it can be observed that $C<\infty$ and $b>0$ exist such that for any $l \geq 0$ and $y \in \partial \mathcal{B}(w(0))$,

$$
\begin{aligned}
& P^{y}\left(\rho_{l}=\frac{n^{2}}{4}\right) \\
\leq & \sup _{l \geq 0} \sup _{y \in \partial \mathcal{B}(w(0))} P^{y}\left(C_{1, n, l}\right) \sup _{l \geq 0} \sup _{y \in \partial \mathcal{B}(w(1))} P^{y}\left(C_{2, n, l}\right) \ldots \sup _{l \geq 0} \sup _{y \in \partial \mathcal{B}\left(w\left(n^{2}-1\right)\right)} P^{y}\left(C_{n^{2} / 4, n, l}\right) \\
\leq & C \exp \left(-b n^{2}\right) .
\end{aligned}
$$

Using the definition of $h_{n}$, we see that for any $l \geq 0, y$ with $D \leq 2^{-n-1}$

$$
P^{y}\left(L_{1, l}\right) \geq P^{y}\left(L_{1, l} \cap\left\{\rho_{l}<\frac{n^{2}}{4}\right\}\right) \geq h_{n} P^{y}\left(A_{1, l} \cap\left\{\rho_{l}<\frac{n^{2}}{4}\right\}\right) .
$$

However, we obtain

$$
P^{y}\left(A_{1, l} \cap\left\{\rho_{l}<\frac{n^{2}}{4}\right\}\right) \geq P^{y}\left(A_{1, l}\right)-C \exp \left(-b n^{2}\right) \geq P^{y}\left(A_{1, l}\right)\left(1-C \exp \left(-b n^{2}\right)\right) .
$$

Therefore, if we set $\delta_{n}=C \exp \left(-b n^{2}\right)$, we complete the proof.

Lemma 3.6. There exists $C<\infty$ such that for any $l \geq 0$ and $n \in \mathbb{N}$

$$
a_{n, l} \leq C a_{n+1, l} .
$$

Proof: The strong Markov property and Lemmas 3.4 yield that for any $x \in \partial \mathcal{B}$

$$
\begin{aligned}
P^{x}\left(\left(B+F_{l}\right)\left[0, \tau\left(e^{n}, l\right)\right] \subset W[\alpha, \beta]\right) & \leq\left[1_{A(n-1, l)} P^{B\left(\tau_{n-1, l}\right)}\left(A_{n, l}\right)\right] \\
& \leq C E^{x}\left[1_{A(n-1, l)} P^{B\left(\tau_{n-1, l}\right)}\left(L_{n, l}\right)\right] \\
& \leq C P^{x}\left(L_{n, l}\right) .
\end{aligned}
$$

Hence, (3.3) yields

$$
a_{n, l} \leq C \sup _{x \in \partial \mathcal{B}} P^{x}\left(L_{n, l}\right) \times \inf _{x \in \tilde{W} \cap \partial \mathcal{B}} \inf _{l \geq 0} P^{x}\left(A_{1, l} \cap\left\{\left(B+F_{l}\right)\left(\tau_{e, l}\right) \in \tilde{W}\right\}\right) \leq C a_{n+1, l} .
$$

Therefore, the desired result holds.

Lemma 3.7. There exists $C<\infty$ such that for any $l \geq 0, n \in \mathbb{N}$ and $r \geq 1$

$$
\begin{aligned}
& a_{n, l} \leq a\left(n-r, l / e^{2 r}\right), \\
& a_{n, l} \leq C a\left(n, l / e^{2}\right) .
\end{aligned}
$$

Proof: Note that for any $x \in W \cap \partial \mathcal{B}, x^{\prime} \in(x+W) \cap \partial \mathcal{B}\left(e^{r}\right), r \geq 1, y \in \mathbb{R}^{d}$, if $y+x \in$ $W$, then $y+x^{\prime} \in W$. Hence, if $B(s)+F_{l}(s)+x \in W$, then $B(s)+F_{l}(s)+x^{\prime} \in W$ for any $0 \leq s \leq \inf \left\{t \geq 0: B(t)+F_{l}(t)+x \in \partial \mathcal{B}_{e^{n}}\right\}$ and Brownian motion $(B(t))_{t \geq 0}$ starting the origin (see Figure 3.1). Thus, for any $x \in W \cap \partial \mathcal{B}, x^{\prime} \in(x+W) \cap \partial \mathcal{B}\left(e^{r}\right)$, $r \geq 1$, and $l \geq 0$, it is true that $P^{x}\left(A_{n, l}\right) \leq P^{x^{\prime}}\left(A_{n, l}\right)$ given that $\inf \{t \geq 0$ : $\left.B(t)+F_{l}(t)+x \in \partial \mathcal{B}_{e^{n}}\right\} \leq \inf \left\{t \geq 0: B(t)+F_{l}(t)+x^{\prime} \in \partial \mathcal{B}_{e^{n}}\right\}$. In addition, 
the scaling property states that $P^{x^{\prime}}\left(A_{n, l}\right)=P^{x^{\prime} / \exp (r)}\left(A\left(n-r, l / e^{2 r}\right)\right)$. Therefore, from Lemma 3.6, we obtain $a_{n, l} \leq a\left(n-1, l / e^{2}\right) \leq C a\left(n, l / e^{2}\right)$. Consequently, we obtain the desired results.

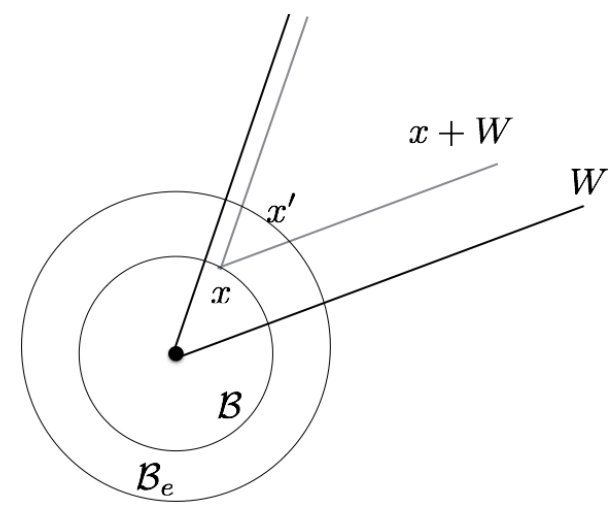

FiguRE 3.1.

Lemma 3.8. There exists $C<\infty$ such that for any $n \in \mathbb{N}$

$$
\sup _{l \in \mathcal{G}_{0}} a_{n, l} \leq C \inf _{x^{\prime} \in \tilde{W} \cap \mathcal{B}} P^{x^{\prime}}\left(A_{n}\right) .
$$

Remark 3.9. Lemma 3.8 yields that there exists $C<\infty$ such that for any $n \in \mathbb{N}$

$$
\sup _{l \in \mathcal{G}_{0}} a_{n, l} \leq C a_{n}
$$

Proof: Let

$$
c_{0}:=\inf \left\{c \geq 0: \min _{x \in W \cap \partial \mathcal{B}} \operatorname{dist}\left(\tilde{W} \cap \partial \mathcal{B}_{c}, \partial(x+W)\right)=K e, \tilde{W} \cap \partial \mathcal{B}_{c} \subset x+W\right\} .
$$

Note that $\max _{t \geq 0} \max _{l \in \mathcal{G}_{0}}\left|F(t)-F_{l}(t)\right|=K e$. Thus, for $x \in \partial \mathcal{B}, x^{\prime} \in \tilde{W} \cap \partial \mathcal{B}\left(c_{0}\right)$, $y \in \mathbb{R}^{d}, t \geq 0$ and $l \in \mathcal{G}_{0}$, if $x+y+F_{l}(t) \in W$, then $x^{\prime}+y+F(t) \in W$ given that $\operatorname{dist}\left(x^{\prime}, x+\partial W\right) \leq K e$. Hence, if $B$ is a Brownian motion starting at the origin, $x \in \partial \mathcal{B}, l \in \mathcal{G}_{0}$, and $x+B(t)+F_{l}(t) \in W$, then $x^{\prime}+B(t)+F(t) \in$ $W$ for any $x^{\prime} \in \tilde{W} \cap \partial \mathcal{B}\left(c_{0}\right)$ (see Figure 3.2). Thus, we have $a\left(n+\log c_{0}, l\right) \leq$ $\inf _{x^{\prime} \in \tilde{W} \cap \partial \mathcal{B}\left(c_{0}\right)} P^{x^{\prime}}\left(A\left(n+\log c_{0}\right)\right)$ given that inf $\left\{t \geq 0: B(t)+F_{l}(t)+x \in \partial \mathcal{B}_{e^{n+c_{0}}}\right\} \leq$ $\inf \left\{t \geq 0: B(t)+F(t)+x^{\prime} \in \partial \mathcal{B}_{e^{n+c_{0}}}\right\}$. Therefore, the scaling property and Lemma 3.6 yield

$$
a_{n, l} \leq C a\left(n+\log c_{0}, l\right) \leq C \inf _{x^{\prime} \in \tilde{W} \cap \partial \mathcal{B}\left(c_{0}\right)} P^{x^{\prime}}\left(A\left(n+\log c_{0}\right)\right)=C \inf _{x \in \tilde{W} \cap \partial \mathcal{B}} P^{x}\left(A_{n}\right) .
$$

Consequently, the desired result holds. 


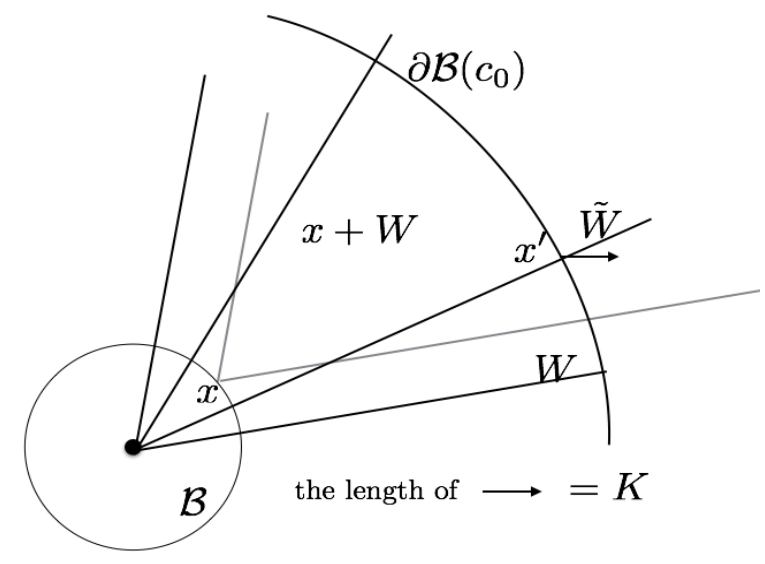

FiguRE 3.2 .

Proof of Theorem 2.1: By the Markov property and the scaling property, we obtain

$$
\begin{aligned}
a_{n+m} \leq & \sup _{x \in \partial \mathcal{B}} P^{x}\left((B+F)\left[0, \tau\left(e^{n}\right)\right] \subset W ; \tau\left(e^{n}\right) e^{-2 n} \in \mathcal{G}_{0}\right) \\
& \times \sup _{x \in \partial \mathcal{B}_{e^{n}}} \sup _{l \geq 0} P^{x}\left(\left(B+F_{l}\right)\left[0, \tau\left(e^{n+m}, l\right)\right] \subset W\right) \\
& +\sum_{s=1}^{\infty} \sup _{x \in \partial \mathcal{B}} P^{x}\left((B+F)\left[0, \tau\left(e^{n}\right)\right] \subset W ; \tau\left(e^{n}\right) e^{-2 n} \in \mathcal{G}_{s}\right) \\
& \times \sup _{x \in \partial \mathcal{B}_{e} n} \sup _{l \in \mathcal{G}_{s}} P^{x}\left(\left(B+F_{l}\right)\left[0, \tau\left(e^{n+m}, l\right)\right] \subset W\right) \\
= & \sup _{x \in \partial \mathcal{B}} P^{x}\left((B+F)\left[0, \tau\left(e^{n}\right)\right] \subset W ; \tau\left(e^{n}\right) e^{-2 n} \in \mathcal{G}_{0}\right) \\
& \times \sup _{x \in \partial \mathcal{B}} \sup _{l \geq 0} P^{x}\left(\left(B+F_{l}\right)\left[0, \tau\left(e^{m}, l\right)\right] \subset W\right) \\
& +\sum_{s=1}^{\infty} \sup _{x \in \partial \mathcal{B}} P^{x}\left((B+F)\left[0, \tau\left(e^{n}\right)\right] \subset W ; \tau\left(e^{n}\right) e^{-2 n} \in \mathcal{G}_{s}\right) \\
& \times \sup _{x \in \partial \mathcal{B}} \sup _{l \in \mathcal{G}_{s}} P^{x}\left(\left(B+F_{l}\right)\left[0, \tau\left(e^{m}, l\right)\right] \subset W\right) .
\end{aligned}
$$

Note that if $\tau\left(e^{n}\right) e^{-2 n} \geq e^{s}, \tau\left(e^{j}, l\right)-\tau\left(e^{j-1}, l\right) \geq e^{-n+j-1} e^{s}\left(e^{n}\right)^{2}=e^{s}\left(e^{j}\right)^{2} e^{n-j} / e$ for any $j=1, \ldots, n$. Then, (3.1) yields that there exists $C>0$ such that for any $s>0$

$$
\begin{aligned}
& \sup _{x \in \partial \mathcal{B}} P^{x}\left((B+F)\left[0, \tau\left(e^{n}\right)\right] \subset W ; \tau\left(e^{j}\right)-\tau\left(e^{j-1}\right) \geq \frac{e^{s}}{e}\left(e^{j}\right)^{2} e^{n-j}\right) \\
& \leq a_{j-1} \sup _{l \geq 0} \sup _{x \in \partial \mathcal{B}(\exp (j-1))} P^{x}\left(\tau\left(e^{j}, l\right)-\tau\left(e^{j-1}, l\right) \geq \frac{e^{s}}{e}\left(e^{j}\right)^{2} e^{n-j}\right) \\
& \leq C a_{j-1} \exp \left(-C e^{s} e^{n-j}\right) .
\end{aligned}
$$


Hence, (3.6) yields

$$
\begin{aligned}
& P^{x}\left((B+F)\left[0, \tau\left(e^{n}\right)\right] \subset W ; \tau\left(e^{n}\right) e^{-2 n} \in \mathcal{G}_{s}\right) \\
\leq & \sum_{j=0}^{n} C a_{j-1} \exp \left(-C e^{s} e^{n-j}\right)=a_{n} \sum_{j=0}^{n} C \frac{a_{j-1}}{a_{n}} \exp \left(-C e^{s} e^{n-j}\right) \\
\leq & C \exp \left(-C e^{s}\right) a_{n} .
\end{aligned}
$$

In addition, (3.6) yields that for any $s>0$ there exists $C<\infty$ such that

$$
\begin{aligned}
& \sup _{x \in \partial \mathcal{B}} \sup _{l \in \mathcal{G}_{s}} P^{x}\left(\left(B+F_{l}\right)\left[0, \tau\left(e^{m}, l\right)\right] \subset W\right) \\
& \leq C \sup _{x \in \partial \mathcal{B}} \sup _{l \in \mathcal{G}_{s-1}} P^{x}\left(\left(B+F_{l}\right)\left[0, \tau\left(e^{m}, l\right)\right] \subset W\right) .
\end{aligned}
$$

Consequently, we obtain

$$
\begin{aligned}
& \sum_{s=0}^{\infty} C \exp \left(-C e^{s}\right) \sup _{x \in \partial \mathcal{B}} \sup _{l \in \mathcal{G}_{s}} P^{x}\left(\left(B+F_{l}\right)\left[0, \tau\left(e^{m}, l\right)\right] \subset W\right) \\
\leq & C \sum_{s=0}^{\infty} C \exp \left(-C e^{s}\right) C^{s} \sup _{x \in \partial \mathcal{B}} \sup _{l \in \mathcal{G}_{0}} P^{x}\left(\left(B+F_{l}\right)\left[0, \tau\left(e^{m}, l\right)\right] \subset W\right) \\
\leq & C \sup _{x \in \partial \mathcal{B}} \sup _{l \in \mathcal{G}_{0}} P^{x}\left(\left(B+F_{l}\right)\left[0, \tau\left(e^{m}, l\right)\right] \subset W\right) .
\end{aligned}
$$

Therefore, by Remark 3.9 we obtain the desired result.

\subsection{Proof of Theorem 2.2.}

Lemma 3.10. There exist $g>0$ and $C<\infty$ such that for any $n \in \mathbb{N}$

$$
a_{n} \leq C a_{n, g} .
$$

Proof: We pick $g$ such that

$$
K g^{1 / 2}\left(=\max _{t \geq 0}\left|F(t)-F_{g}(t)\right|\right)=\min _{x \in W \cap \partial \mathcal{B}} \max _{\left(x_{1}^{\prime}, \ldots, x_{d}^{\prime}\right) \in(x+W) \cap \partial \mathcal{B}_{e}\left(z_{1}, \ldots, z_{d}\right) \in \partial W}\left|x_{1}^{\prime}-z_{1}\right| .
$$

Note that for any $y \in \mathbb{R}^{d}, x \in W \cap \partial \mathcal{B}$ and $x^{\prime} \in(x+W) \cap \partial \mathcal{B}_{e}$, if $y+F(s)+x \in W$, then $y+F_{g}(s)+x^{\prime} \in W$. Thus, if $B(s)+F(s)+x \in W$, then $B(s)+F_{g}(s)+x^{\prime} \in W$ for any $0 \leq s \leq \inf \left\{t \geq 0: B(t)+F(t)+x \in \partial \mathcal{B}_{e^{n}}\right\}$ and Brownian motion $(B(t))_{t \geq 0}$ starting the origin (see Figure 3.3). Hence, for any $x \in W \cap \partial \mathcal{B}$ there exists $x^{\prime} \in W \cap \partial \mathcal{B}_{e}$ such that $P^{x}\left(A_{n}\right) \leq P^{x^{\prime}}\left(A_{n, g}\right)$. Therefore, (3.5) and Lemma 3.6 yield that there exists $C<\infty$ such that for any $n \in \mathbb{N}$, and $x \in W \cap \partial \mathcal{B}, P^{x}\left(A_{n}\right) \leq$ $a\left(n-1, g / e^{2}\right) \leq C a_{n, g}$ holds.

To show Theorem 2.2, we introduce the following lemma.

Lemma 3.11. For any $b^{\prime}>0$ and $b>0$ there exists $C<\infty$ such that for any $n \in \mathbb{N}$

$$
a_{n} \leq C \inf _{x \in \tilde{W} \cap \partial \mathcal{B}} \inf _{b^{\prime} \leq l \leq b} P^{x}\left(A_{n, l}\right) .
$$

Proof: Note that for any $b^{\prime}>0$ and $b>0$ there exists $c>0$ such that

$$
\inf _{a \in \partial \mathcal{B}} \inf _{b^{\prime} \leq l \leq b} \inf _{x \in \tilde{W} \cap \partial \mathcal{B}(\sqrt{g / 2 l})} P^{x}\left(\left(B+F_{g / 2}\right)\left[0, \frac{g}{2}\right] \subset W ;\left(B+F_{g / 2}\right)\left(\frac{g}{2}\right) \in a+(W \cap \mathcal{B})\right)>c .
$$




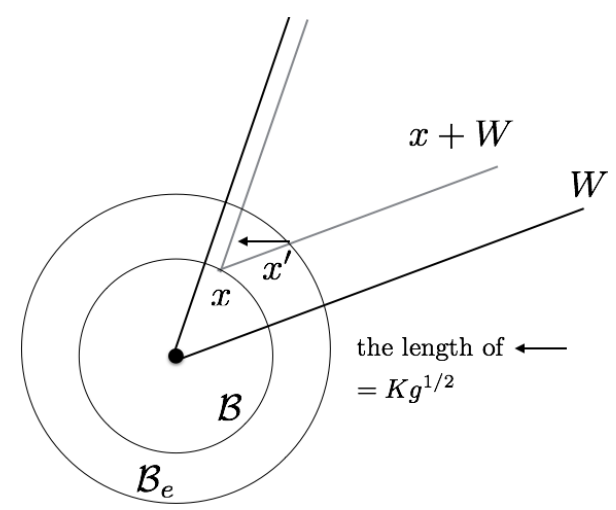

FIGURE 3.3 .

In addition, we have

$$
\sup _{x \in \partial \mathcal{B}} P^{x}\left(A_{n, g}\right) \leq \sup _{a \in \partial \mathcal{B}} \inf _{x^{\prime} \in a+(W \cap \mathcal{B})} P^{x^{\prime}}\left(A_{n, g}\right) .
$$

Because if $B(s)+F_{g}(s)+x \in W$, then there exists $a \in \partial \mathcal{B}$ such that $B(s)+F_{g}(s)+$ $a \in W$ for any $0 \leq s \leq \inf \left\{t \geq 0: B(t)+F_{g}(t)+x \in \partial \mathcal{B}_{e^{n}}\right\}$ and Brownian motion $(B(t))_{t \geq 0}$ starting the origin and hence for any $y \in W \cap \mathcal{B}$ there exists $a \in \partial \mathcal{B}$ such that $B(s)+F_{g}(s)+y+a \in W$. Then, we obtain

$$
\begin{aligned}
& \sup _{x \in \partial \mathcal{B}} P^{x}\left(A_{n, g}\right) \\
\leq C & \sup _{a \in \partial \mathcal{B}} \inf _{x \in a+(W \cap \mathcal{B})} P^{x}\left(A_{n, g}\right) \\
\times & \inf _{a \in \partial \mathcal{B}} \inf _{b^{\prime} \leq l \leq b} \inf _{x \in \tilde{W} \cap \partial \mathcal{B}(\sqrt{g / 2 l})} P^{x}\left(\left(B+F_{g / 2}\right)\left[0, \frac{g}{2}\right] \subset W ;\left(B+F_{g / 2}\right)\left(\frac{g}{2}\right) \in a+(W \cap \mathcal{B})\right) \\
\leq C & \sup _{a \in \partial \mathcal{B}}\left\{\inf _{b^{\prime} \leq l \leq b} \inf _{x \in \tilde{W} \cap \partial \mathcal{B}(\sqrt{g / 2 l})} P^{x}\left(\left(B+F_{g / 2}\right)\left[0, \frac{g}{2}\right] \subset W ;\left(B+F_{g / 2}\right)\left(\frac{g}{2}\right) \in a+(W \cap \mathcal{B})\right)\right. \\
\times & \left.\inf _{x \in a+(W \cap \mathcal{B})} P^{x}\left(A_{n, g}\right)\right\} \\
\leq C & \sup _{a \in \partial \mathcal{B}}\left\{\inf _{b^{\prime} \leq l \leq b} \inf _{x \in \tilde{W} \cap \partial \mathcal{B}(\sqrt{g / 2 l})} P^{x}\left(\left(B+F_{g / 2}\right)\left[0, \tau_{n, g / 2}\right] \subset W\right)\right\} \\
= & C \inf _{b^{\prime} \leq l \leq b} \inf _{x \in \tilde{W} \cap \partial \mathcal{B}(\sqrt{g / 2 l})} P^{x}\left(\left(B+F_{g / 2}\right)\left[0, \tau_{n, g / 2}\right] \subset W\right) \\
\leq C & \inf _{x \in \tilde{W} \cap \partial \mathcal{B}} \inf _{b^{\prime} \leq l \leq b} P^{x}\left(\left(B+F_{l}\right)\left[0, \tau_{n-2 l / g, l}\right] \subset W\right) .
\end{aligned}
$$

Finally, by Lemma 3.5 and the same argument as Lemma 3.6, it is bound by

$$
C \inf _{x \in \tilde{W} \cap \partial \mathcal{B}} \inf _{b^{\prime} \leq l \leq b} P^{x}\left(\left(B+F_{l}\right)\left[0, \tau_{n, l}\right] \subset W\right) .
$$

Therefore, by Lemma 3.10 we obtain the desired result. 
Lemma 3.12. There exist $C<\infty$ and $c>0$ such that for any $n \in \mathbb{N}$ and $0<b<\infty$

$$
\begin{aligned}
& \sup _{x \in \partial \mathcal{B}} P^{x}\left(A_{n} \cap\left\{\tau\left(e^{n}\right) e^{-2 n} \geq b\right\}\right) \leq C e^{-c b} a_{n}, \\
& \sup _{x \in \partial \mathcal{B}} P^{x}\left(A_{n} \cap\left\{\tau\left(e^{n}\right) e^{-2 n} \leq b\right\}\right) \leq C e^{-c / b} a_{n} .
\end{aligned}
$$

Proof: Lemma 3.6 and (3.2) yield

$$
\begin{aligned}
& \sup _{x \in \partial \mathcal{B}} P^{x}\left(A_{n} \cap\left\{\tau\left(e^{n}\right) e^{-2 n} \leq b\right\}\right) \\
\leq & \sup _{x \in \partial \mathcal{B}} P^{x}\left(A_{n-1}\right) \sup _{l \geq 0} \sup _{y \in \partial \mathcal{B}(n-1)} P^{y}\left(\tau\left(e^{n}\right) e^{-2 n} \leq b\right) \leq C e^{-c / b} a_{n} .
\end{aligned}
$$

Then, we obtain (3.9). In addition (3.7) and Corollary 2.4 yield (3.8).

Proof of Theorem 2.2: First, Lemma 3.5 and (3.8) yield that there exists $C<\infty$ such that for any $n \in \mathbb{N}$

$$
\begin{aligned}
\sup _{x \in \partial \mathcal{B}} P^{x}\left((B+F)\left[0, \tau\left(e^{n}\right)\right] \subset W ; \tau\left(e^{n}\right) e^{-2 n} \geq b\right) & \leq C e^{-c b} a_{n} \\
& \leq C e^{-c b} \sup _{x \in \partial \mathcal{B}} P^{x}\left(L_{n, 0}\right) .
\end{aligned}
$$

In addition, (3.9) yields

$$
\begin{aligned}
& \sup _{x \in \partial \mathcal{B}} P^{x}\left((B+F)\left[0, \tau\left(e^{n}\right)\right] \subset W ; \tau\left(e^{n}\right) e^{-2 n} \leq b^{\prime}\right) \\
& \leq C e^{-c / b^{\prime}} a_{n} \leq C e^{-c / b^{\prime}} \sup _{x \in \partial \mathcal{B}} P^{x}\left(L_{n, 0}\right) .
\end{aligned}
$$

If we pick sufficiently small $b^{\prime}$ and large $b$, by Lemma 3.4 there exists $c>0$ such that for any $n \in \mathbb{N}$

$$
\sup _{x \in \partial \mathcal{B}} P^{x}\left(L_{n, 0} \cap\left\{\tau\left(e^{n}\right) e^{-2 n} \in\left[b^{\prime}, b\right]\right\}\right) \geq c \sup _{x \in \partial \mathcal{B}} P^{x}\left(L_{n, 0}\right) \geq c \sup _{x \in \partial \mathcal{B}} P^{x}\left(A_{n}\right) .
$$

Consequently,

$$
\begin{aligned}
a_{n+m} & \geq \sup _{x \in \partial \mathcal{B}} P^{x}\left(L_{n, 0} \cap\left\{\tau\left(e^{n}\right) e^{-2 n} \in\left[b^{\prime}, b\right]\right\}\right) \inf _{x \in \tilde{W} \cap \partial \mathcal{B}(\exp (n))} \inf _{b^{\prime} \leq l \leq b} P^{x}\left(A_{m, l}\right) \\
& \geq c a_{n} \inf _{x \in \tilde{W} \cap \partial \mathcal{B} b^{\prime} \leq l \leq b} \inf ^{x}\left(A_{m, l}\right) .
\end{aligned}
$$

Therefore, from Lemma 3.11, we obtain the desired result.

Now, we show (2.1) in Corollary 2.4.

Proof of (2.2) in Corollary 2.4: Note that Fekete's lemma holds: if $\left\{b_{n}\right\}_{n=1}^{\infty}$ is a superadditive (subadditive) sequence with nonpositive elements, $\left(b_{n} / n\right)$ is bounded below and converges to $\sup \left\{b_{n} / n: n \in \mathbb{N}\right\}$ (or $\left.\inf \left\{b_{n} / n: n \in \mathbb{N}\right\}\right)$. If we set $\tilde{a}_{n}:=\log \left(a_{n} / C\right)$, then $\left\{\tilde{a}_{n}\right\}_{n=1}^{\infty}$ is a superadditive sequence with nonpositive terms. Thus, Fekete's lemma implies that there exist $C<\infty$ and $\xi>0$ such that $\tilde{a}_{n} \leq-\xi n$ and hence $a_{n} \leq C e^{-\xi n}$ with the aid of Theorem 2.2. By Lemma 3.4, we obtain $\xi<\infty$. Therefore, given that $P^{x}\left((B+F)\left[0, \tau_{n}\right] \subset W(\alpha, \beta)\right)$ is decreasing on $n$, we obtain (2.2). 
Proof of (2.1) in Corollary 2.4: Fekete's lemma implies that if there exist $C>0$ such that for any $n, m \in \mathbb{N}, a_{n} \leq C a_{n} a_{m}$, then there exists $c>0$ such that $a_{n} \geq$ $c e^{-\xi n}$ with the same argument as the proof of (2.2) in Corollary 2.4. Theorem 2.1 states that there exists $c>0$ such that for any $n \in \mathbb{N}$, and $x \in \tilde{W} \cap \partial \mathcal{B}$

$$
c n^{-\xi} \leq \sup _{x \in \partial \mathcal{B}} P^{x}\left((B+F)\left[0, \tau_{n}\right] \subset W\right) .
$$

Subsequently, Lemma 3.8 yields the desired result.

\subsection{Proof of Corollary 2.5.}

Proof of Corollary 2.5: First, we show the first claim. Since we emphasize on $K$ in $F(t)$, we write $A_{n}(K)$ for $A_{n}$. Let $\xi_{1}(K):=\sup _{K^{\prime} \leq K} \xi\left(K^{\prime}\right)$. Based on Remark 2.3, Theorem 2.2 and Fekete's lemma state that there exists $C<\infty$ such that for any $K \leq 1$, and $x \in \partial \mathcal{B}$

$$
P^{x}\left(A_{n+1}(0)\right) \leq P^{x}\left(\cap_{K^{\prime} \leq K} A_{n}\left(K^{\prime}\right)\right) \leq \min _{K^{\prime} \leq K} P^{x}\left(A_{n}\left(K^{\prime}\right)\right) \leq C e^{-\xi_{1}(K) n} .
$$

Since $\xi_{1}(K) \geq \xi(0)$, we obtain $\xi_{1}(K) \rightarrow \xi(0)$ as $K \rightarrow 0$. To show the other inequality, let $\xi_{2}(K):=\inf _{K^{\prime} \leq K} \xi\left(K^{\prime}\right)$. By Remark 2.3, Theorem 2.1 and Fekete's lemma yield that there exists $C<\infty$ such that for any $K \leq 1$ and $x \in \partial \mathcal{B}$,

$$
P^{x}\left(A_{n+1}(0)\right) \geq P^{x}\left(\cup_{K^{\prime} \leq K} A_{n}\left(K^{\prime}\right)\right) \geq \max _{K^{\prime} \leq K} P^{x}\left(A_{n}\left(K^{\prime}\right)\right) \geq C e^{-\xi_{2}(K) n} .
$$

Since $\xi_{2}(K) \leq \xi(0)$, we obtain $\xi_{2}(K) \rightarrow \xi(0)$ as $K \rightarrow 0$. Therefore, we obtain the first result.

Next, we show the second claim. By monotonicity, the desired result is obtained when $\beta=(1,0)$ or $(1,0,0)$ and $\alpha$ is an acute angle. Let $C_{1}(K):=$ $\inf _{x \in \tilde{W} \cap \partial \mathcal{B}} \inf _{l \leq 1} P\left(L_{e, l} \cap\left\{\tau_{e, l} \leq e^{2}-1\right\}\right)$. It is trivial that as $K \rightarrow \infty$,

$$
C_{1}(K) \rightarrow 1 \text {. }
$$

In addition, for any $x \in \tilde{W} \cap \partial \mathcal{B}$ and $n \in \mathbb{N}$

$$
P^{x}\left(A_{n}(K)\right) \geq C_{1}(K)^{n} .
$$

Hence, we obtain the second claim.

Next, we show the third claim. Choose sufficiently large $M<\infty$ such that for any $v \in \mathbb{N}$,

$$
\lim _{K \rightarrow \infty} \sup _{x \in \partial \mathcal{B}} \sup _{l \leq M^{2 v}} P^{x}\left(A_{\log M, l}(K)\right)=0 .
$$

The large deviation estimate states that $C<\infty$ and $c>0$ exist such that for any $s<\infty$ and $M<\infty$,

$$
\sup _{K \geq 0, l \geq 0} \sup _{x \in \partial \mathcal{B}} P^{x}\left(\tau_{M, l} M^{-2} \geq s\right) \leq C \exp (-c s) .
$$

Next, fix $\epsilon>0$ and choose sufficiently large $v \in \mathbb{N}$ such that

$$
\sum_{s=v-1}^{\infty} \frac{C \exp \left(-c^{\prime} M^{2 s}\right)}{\epsilon^{s+1}} \leq \frac{1}{4}
$$

In addition, based on (3.10), we can choose $K<\infty$ such that

$$
a_{n}^{\prime}:=\sup _{K^{\prime} \geq K} \sup _{x \in \partial \mathcal{B}} \sup _{l \leq M^{2 v}} P^{x}\left(A_{\log M, l}\left(K^{\prime}\right)\right)<\epsilon .
$$


Note that (3.5) yields

$$
\sup _{x \in \partial \mathcal{B}} P^{x}\left(A_{n, l}\right) \leq \sup _{x \in \partial \mathcal{B}} P^{x}\left(A\left(n-1, l / e^{2}\right)\right) .
$$

Then, by (3.11) we obtain

$$
\begin{aligned}
a_{n+1}^{\prime} \leq & \sup _{K^{\prime} \geq K} \sup _{x \in \partial \mathcal{B}} \sup _{l \leq M^{2 v}} P^{x}\left(\left(B+F_{l}\right)\left(0, \tau\left(M^{n+1}, l\right)\right) \subset W ; \tau_{M, l} M^{-2} \leq M^{2 v}-M^{2 v-2}\right) \\
& +\sum_{s=v-1}^{n-2} \sup _{K^{\prime} \geq K} \sup _{x \in \partial \mathcal{B}} \sup _{l \leq M^{2 v}} P^{x}\left(\left(B+F_{l}\right)\left(0, \tau\left(M^{n+1}, l\right)\right) \subset W\right. \\
& \left.\tau_{M, l} M^{-2} \in\left[M^{2 s}-M^{2 v-2}, M^{2 s+2}-M^{2 v-2}\right]\right) \\
& +\sup _{K^{\prime} \geq K} \sup _{x \in \partial \mathcal{B}} \sup _{l \leq M^{2 v}} P^{x}\left(\tau_{M, l} M^{-2} \geq M^{2 n}\right) \\
& \leq \epsilon a_{n}^{\prime}+\sum_{s=v-1}^{n} C \exp \left(-c M^{2 s}\right) a_{n-s}^{\prime} .
\end{aligned}
$$

Hence, if we set $b_{n}^{\prime}=a_{n}^{\prime} / \epsilon^{n}$, we obtain that for any $m \geq v$

$$
b_{m+1}^{\prime}-b_{m}^{\prime} \leq \sum_{s=v-1}^{m} C \frac{\exp \left(-c^{\prime} M^{2 s}\right)}{\epsilon^{s+1}} b_{m-s}^{\prime} .
$$

Summing over $m=v, v+1, \ldots, n$, by (3.12) we obtain that for any $n \geq v$

$$
b_{n+1}^{\prime} \leq b_{v}^{\prime}+\sum_{m=0}^{n-v} \frac{b_{m}^{\prime}}{4} \leq 2 \sum_{m=0}^{n} b_{m}^{\prime} .
$$

Then, the induction yields that for any $\epsilon>0$ there exists $C<\infty$ such that for any $n \in \mathbb{N}$

$$
b_{n}^{\prime} \leq C 3^{n}
$$

and, hence,

$$
\limsup _{n \rightarrow \infty} \frac{\log a_{n}^{\prime}}{n} \leq \log \epsilon+3 .
$$

Therefore, we have the desired result.

\subsection{Proof of Corollary 2.6.}

Proof of Corollary 2.6: By monotonicity, it suffices to show that there exist $0<$ $C_{1}, C_{2}<\infty$ such that for any $n \in \mathbb{N}$ and $x \in \tilde{W} \cap \partial \mathcal{B}$,

$$
C_{1} e^{-\xi n} \leq P^{x}\left((B+F)\left[0, e^{2 n}\right] \subset W[\alpha, \beta]\right) \leq C_{2} e^{-\xi n} .
$$

Equations (2.2) and (3.9) conclude that for any $b<\infty$,

$$
\begin{aligned}
& \sup _{x \in \tilde{W} \cap \partial \mathcal{B}} P^{x}\left((B+F)\left[0, e^{2 n}\right] \subset W\right) \\
\geq & \sup _{x \in \tilde{W} \cap \partial \mathcal{B}} P^{x}\left((B+F)\left[0, \tau\left(b e^{n}\right)\right] \subset W\right) \\
& -\sup _{x \in W \cap \partial \mathcal{B}} P^{x}\left((B+F)\left[0, \tau\left(b e^{n}\right)\right] \subset W ; \tau_{b e^{n}} e^{-2 n} \leq 1\right) \\
\geq & \left(b e^{n}\right)^{-\xi}-C\left(b e^{n}\right)^{-\xi} e^{-c b^{2}} .
\end{aligned}
$$


Next, if we choose sufficiently large $b<\infty$, the last expression is greater than $\left(b e^{n}\right)^{-\xi} / 2$. Hence, we obtain the desired lower bound. We now show the upper bound. Note that for any $x \in W \cap \partial \mathcal{B}$

$$
\begin{aligned}
& \sup _{x \in W \cap \partial \mathcal{B}} P^{x}\left((B+F)\left[0, e^{2 n}\right] \subset W\right) \\
\leq & \sup _{x \in W \cap \partial \mathcal{B}} P^{x}\left((B+F)\left[0, e^{2 n}\right] \subset W\right) \\
\leq & \sup _{x \in W \cap \partial \mathcal{B}} P^{x}\left(A_{n}\right)+\sum_{j=1}^{n} \sup _{x \in W \cap \partial \mathcal{B}} P^{x}\left(A_{j-1} \cap\left\{\tau\left(e^{j-1}\right) \geq e^{2 n}\right\}\right) .
\end{aligned}
$$

Equations (2.2) and (3.8) yield that there exist $C<\infty$ and $c>0$ such that

$$
\begin{aligned}
\sup _{x \in W \cap \partial \mathcal{B}} P^{x}\left(A_{j-1} \cap\left\{\tau\left(e^{j-1}\right) \geq e^{2 n}\right\}\right) & \leq C\left(e^{j-1}\right)^{-\xi} \exp \left(-c e^{n-j}\right) \\
& =C\left(e^{n-1}\right)^{-\xi}\left(e^{n-j}\right)^{\xi} \exp \left(-c e^{n-j}\right) .
\end{aligned}
$$

By summing over $j$, we have the desired upper bound.

\section{References}

T. Antunović, K. Burdzy, Y. Peres and J. Ruscher. Isolated zeros for Brownian motion with variable drift. Electron. J. Probab. 16, no. 65, 1793-1814 (2011). MR2842087.

M. T. Barlow and E. A. Perkins. Brownian motion at a slow point. Trans. Amer. Math. Soc. 296 (2), 741-775 (1986). MR846605.

K. Burdzy. Brownian paths and cones. Ann. Probab. 13 (3), 1006-1010 (1985). MR799436.

B. Davis and E. Perkins. Brownian slow points: the critical case. Ann. Probab. 13 (3), 779-803 (1985). MR799422.

S. N. Evans. On the Hausdorff dimension of Brownian cone points. Math. Proc. Cambridge Philos. Soc. 98 (2), 343-353 (1985). MR795899.

R. Garbit and K. Raschel. On the exit time from a cone for Brownian motion with drift. Electron. J. Probab. 19, no. 63, 27 (2014). MR3238783.

G. F. Lawler. Nonintersecting planar Brownian motions. Math. Phys. Electron. J. 1, Paper 4, approx. 35 (1995). MR1359459.

G. F. Lawler. Hausdorff dimension of cut points for Brownian motion. Electron. J. Probab. 1, no. 2, approx. 20 pp. (1996). MR1386294.

G. F. Lawler. Geometric and fractal properties of Brownian motion and random walk paths in two and three dimensions. In Random walks (Budapest, 1998), volume 9 of Bolyai Soc. Math. Stud., pages 219-258. János Bolyai Math. Soc., Budapest (1999). MR1752896.

G. F. Lawler and B. Vermesi. Fast convergence to an invariant measure for nonintersecting 3-dimensional Brownian paths. ALEA Lat. Am. J. Probab. Math. Stat. 9 (2), 717-738 (2012). MR3069382.

J.-F. Le Gall and T. Meyre. Points cônes du mouvement brownien plan, le cas critique. Probab. Theory Related Fields 93 (2), 231-247 (1992). MR1176726.

P. Mörters and Y. Peres. Brownian motion, volume 30 of Cambridge Series in Statistical and Probabilistic Mathematics. Cambridge University Press, Cambridge (2010). ISBN 978-0-521-76018-8. MR2604525. 
Y. Peres and P. Sousi. Brownian motion with variable drift: 0-1 laws, hitting probabilities and Hausdorff dimension. Math. Proc. Cambridge Philos. Soc. 153 (2), 215-234 (2012). MR2981924.

M. Shimura. Excursions in a cone for two-dimensional Brownian motion. J. Math. Kyoto Univ. 25 (3), 433-443 (1985). MR807490. 